HOST-VIRAL INTERACTIONS

\section{Hosting encounters}

Cell Host Microbe

doi: 10.1016/j.chom.2014.11.002

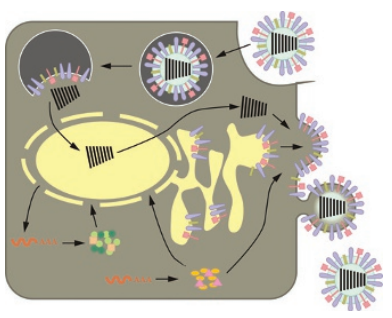

Considering the limited repertoire of proteins produced by viruses as well as the high susceptibility of viral protein targets to develop drug resistance, recent antiviral drug development strategies have focused on targeting host proteins that are essential for viral replication. However, few functional analyses are available for defining relevant host-viral interactions necessary for the viral life cycle. Using interaction with host factors as a proxy for functional significance, Watanabe et al. performed a systematic biochemical analysis of interactions between viral and host proteins. Each of the 11 influenza proteins was tagged and expressed in a human cell line permissive for viral replication. Immunoprecipitation of each viral protein detected a combined 1,292 interactions with host proteins. In a secondary RNAi-based screen, the authors were able to narrow these down to 323 host genes whose decreased expression either increased or reduced viral titers. Gene ontology and pathway analyses led to a network of virus-host protein interactions with potential roles in viral replication among these hits. Among the 91 host factors with the greatest effect on viral replication upon knockdown, they identified 9 that are critical for viral genome replication and transcription, 15 involved in virion formation, 28 involved in incorporation of viral ribonucleoprotein (vRNP) complexes into progeny virions and 23 involved in early steps of the life cycle, including virus-host cell binding, internalization and transport of vRNP complexes to the nucleus. The authors next tested 11 drugs with known targets among the 299 host factors whose downregulation most inhibited viral replication, identifying several candidates that have not been proposed previously as antiviral targets, including JAK1 and a guanine nucleotide exchange factor, GBF1. This rich catalog

\section{GLYCOSYLATION}

\section{A new regulator}

EMBO J. doi:10.15252/embj.201488375
$\mathrm{N}$-linked glycosylation and subsequent tailoring steps of the attached glycans occur in the endoplasmic reticulum and Golgi, mediated by a series of glycosyltransferases and glycosidases. Some of the glycosyltransferases involved are also secreted, and recent work implicated $\gamma$-secretase as the protease initiating secretion, but the full details of this process and the functional importance of secretion are unknown. Voss et al. now identify signal peptide peptidase-like 3 (SPPL3) as the key protease in this process, linking glycosyltransferase secretion to reduced enzyme function. SPPL3 was expected to function similarly to its better-characterized family members such as SPP, but identifying substrates has proven challenging. The authors first observed that inducible expression of SPPL3, but not an inactive mutant, in HEK293 cells reduced the molecular weight of representative glycoproteins; in contrast, SPPL3 knockdown resulted in increased weight. Treatment of the cells with glycosidases specific to O- or N-linked modifications defined SPPL3-induced changes as associated with N-linked glycosylation, whereas treatment with an inhibitor of $\alpha$-mannosidase I-an enzyme involved in $\mathrm{N}$-glycan tailoringindicated that SPPL3's impact was downstream of this activity. Analysis of Sppl3-deficient mice recapitulated the cellular experiments, confirming the link between SPPL3 and $\mathrm{N}$-glycosylation. A comparison of cell supernatants and lysates pointed to GnT-V as the primary substrate of SPPL3, with other $\mathrm{N}$-glycan-modifying enzymes $\beta 3 \mathrm{GnT} 1$ and $\beta 4 \mathrm{GalT} 1$ serving as substrates to a lesser extent. Analytical characterization of glycans excised from proteins supported these results, demonstrating that the branched glycan chains normally generated by GnT-V were strongly diminished in cells overexpressing SPPL3. Finally, the authors confirmed that GnT-V secretion is dependent on proteolysis between Leu29 and Thr33 and in a $\gamma$-secretase-independent manner, as a secretase inhibitor did not alter GnT-V localization. Although the direct proteolysis of the glycosyltransferases by SPPL3 remains to be shown, these results set the stage for further investigations of this important system. opens new avenues to explore critical hostviral interactions at a mechanistic level and to find new antiviral targets.

BIOMATERIALS

\section{Lectins lay the groundwork PLOS ONE 9, el12326 (2014)}

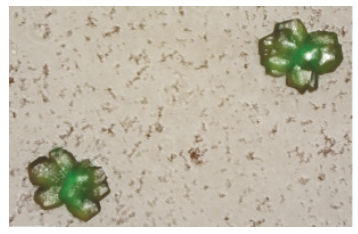

Biomineralization can be described as the controlled crystallization of metals by biological molecules into materials such as shells, bone and diatom cell walls. The crystal structures present in these materials, such as calcite and aragonite, can often be identified. However, defining the biomolecules present in or near the crystals, much less establishing their role in crystallization, is a more challenging task, as the comparatively delicate biomolecules are often damaged by any manipulation to or chemical treatment of the crystals. Biomolecules known to be involved in biomineralization in pearl oyster include the polysaccharide chitin and proteins rich in $\beta$-sheets and acidic residues. Naganuma et al. had previously identified a lectin, PPL1, in Pteria penguin pearl shells with a potential role in biodefense. However, the authors suspected that other lectins could be present, and given that glycans are present in the shell matrix, the authors further suspected that lectins could have a role in mineralization. To test these ideas, the authors returned to their previous affinity chromatography workflow, pulling out three additional lectins, PPL2A, PPL2B and PPL2C. These proteins are the first invertebrate homologs to the jacalinrelated lectins (JRLs), though their substrate specificities diverge from both known JRL preferences and each other: PPL2A's activity in a hemagglutination assay was strongly inhibited by isomaltose and trehalose, whereas PPL2B's activity was affected by larger substrates such as sialoglycoproteins. Interestingly, both trehalose and PPL2A were found to be abundant in the P. penguin mantle-where mineralization occurs-and the nacreous (iridescent) layer. Finally, knockdown of PPL2A but not PPL1 caused defects in shell formation during $P$. penguin larva development, and both PPL2A and PPL2B altered the morphological populations of calcium carbonate crystals in vitro. A more detailed understanding of this interesting system awaits further study. 